\title{
Phononenphysik mit supraleitenden Tunnelkontakten
}

Die Ausbreitung mechanischer Wellen in Festkörpern erfolgt mit der Schallgeschwindigkeit von etwa 1000 bis $10000 \mathrm{~ms}$. Zwischen dieser Geschwindigkeit $c_{;}$, der Frequenz $v$ und der Wellenlänge $\lambda$ besteht die Beziehung:

$c_{s}=y \cdot \lambda$.

Diese Gleichung gilt analog zu den elektromagnetischen Wellen, wobei sich $c_{s}$ und $d i c$ Lichtgeschwindigkeit entsprechen.

Während der obige Zusammenhang für elektromagnetische Wellen bis zu beliebig hohen Frequenzen, d.h. bis zu beliebig kleinen Wellenlängen gilt, gibt es für mechanische Wellen in Festkörpern eine kleinste Wellenlänge. Sie ist durch die atomare Struktur der Materie bedingt: $\lambda_{\text {min }} \approx 2 \mathrm{a} \approx$ $10 \AA$, wobei a der Abstand zweier Atom ist. Damit gibt es auch eine höchstmögliche Frequenz $y_{\max }$ :

$v_{\max } \approx \mathrm{c}_{\mathrm{s}} / \lambda_{\min } \approx 10^{13} \mathrm{~Hz}$

Mechanische Wellen mit Frequenzen größer als $v_{\max }$ können sich in Festkörpern nicht ausbreiten.

Im Beitrag ron $K$. Renk: wurden bereits Methoden zur Erzeugung und zum Nathweis höchstfrequenter mechanischer Wellen (auch Gitterschwingungen oder Phononen genannt) erläutert. Weitere Möglichkeiten hierzu werden in diesem Aufsatz behandelt. Zur Erzeugung von Phononen mit Frequenzen größer $1011 \mathrm{~Hz}$ kann man auch die Elektron-Phonon-Wechselwirkung:* in Metallen oder Supraleitern ausnutzen. In Prinzip wird dabei den Elektronen aus einer Batterie Energie zugeführt, die dann als Phononenemission an das Gitter absegeben wird. Phonon-Ubergänge in Supraleitern sind dabei wegen der speziellen energetischen Struktur der Elcktronenzusrände (Energielücke) ron besonderem $\mathrm{In}$ teresse.

"Siehe "Physik in unserer Zeit" 1, 16 (1974).

*In Metallen existicren neben Elektronen auch andere elementare Anregungen, die Phononen. Sie bezeichnen die quantisierten Einheiten der Gitterschwingungen. Stöte $z$ wischen solchen Phononen und Elektronen werden über die Elektron-Phonon-WW vermittelt.
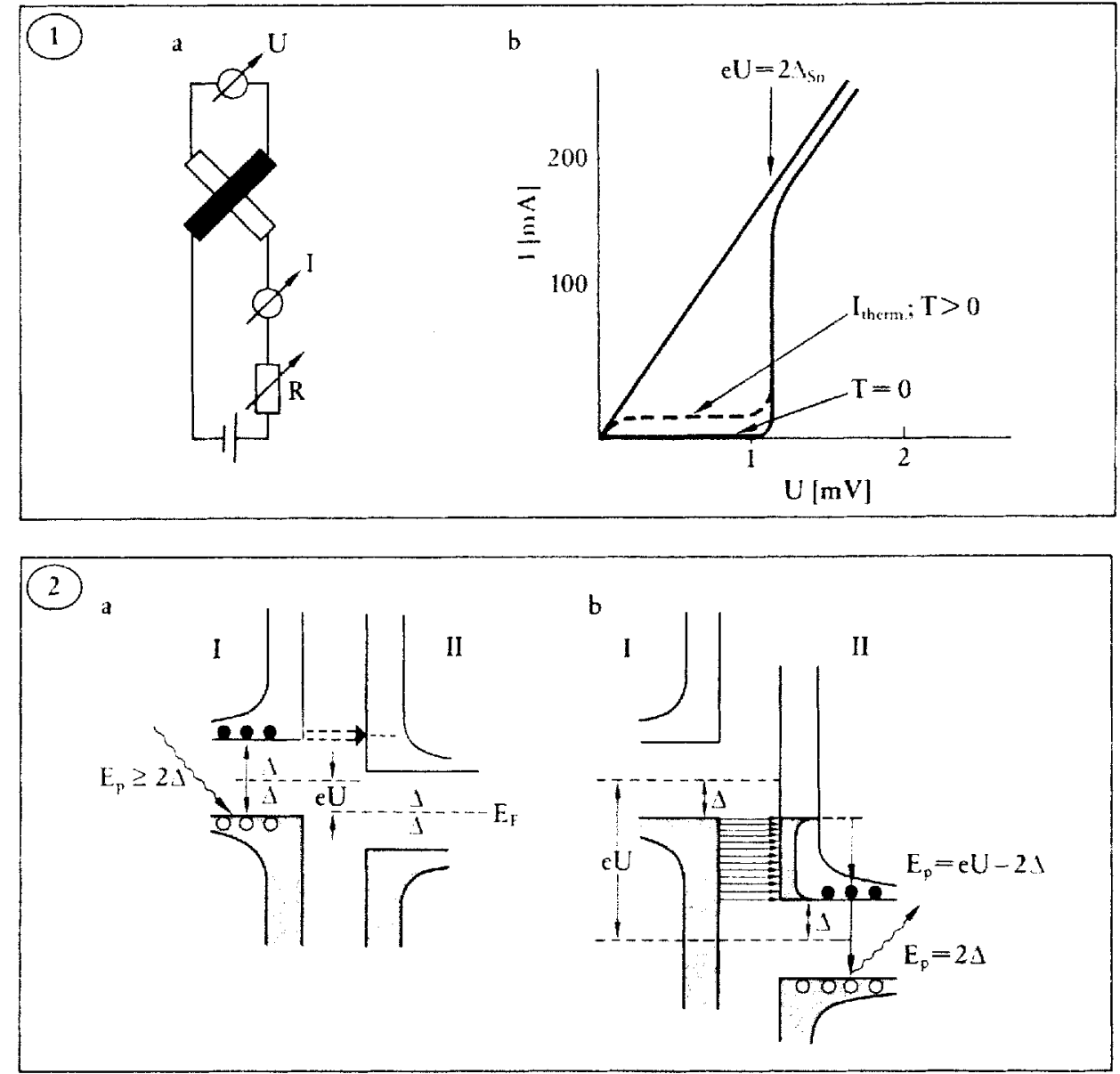

Bei den im folgenden beschriebenen Experimenten zum Phononennachweis und zur Phononendetektion mit Hilfe ron Supraleitern wird zusätzlich noch der quantenmechanische Tunneleffekt ausgenutzt.

Der Tunneleffekt zwischen isolierten Supraleitern

Die Materiewelleneigenschaften von $\mathrm{La}$ dungsträgern erlauben den Elektronenübergang zwischen zwei, durch eine dünne isolierende Oxidschicht getrennten, metallischen Leitern. Diese als Tunneleffekt bezeichnete Erscheinung wurde von Giaever (Nobelpreis 1973) zur direkten Bestimmung der Energielücke der Elektronenanregungen in Supraleitern benutzt. Im Experiment verwendet man, wie in Abbildung 1a skizziert, zwei im Hochvakuum gekreuzt übereinander aufgedampte Schichten eines Supraleiters (z. B. Zinn, Blei oder Aluminium) von etwa 1000 A Dicke. Dic isolierende Trennschicht zwischen den Metallen von etwa 10 bis $20 \AA$ Dicke wird durch Oberflächenoxidation des ersten Aufdampffilmes in Luft oder reinem Sauerstoff hergestellt. Zur Erklärung der in Abbildung $1 \mathrm{~b}$ angege-
Abb. 1. a) Strom - Spannungsmeßanordnung bei einem Tunnelkontakt $\mathrm{U}=$ Spannungsmessung; $\mathrm{J}=$ Strommessung; $\mathrm{R}=$ Vorwiderstand.

b) J-U Charakteristik eines supraleitenden Tunnelkontaktes für $T=0$ sowie für endliche Temperatur $\mathrm{T}>0$.

Abb. 2. Halbleiterschema zur Beschreibung der J-U Charakteristik eines supraleitenden Tunnelkontaktes sowie zur Erklärung der Phonondetektion und -Erzeugung.

a) Thermischer Tunnelstrombereich. $|\mathrm{eU}|$ $<2$ ); $(U=$ Batteriespannung, $\mathrm{e}=$ Elektronenladung). Phononen der Energie $\geqq$ 21 erzeugen zusätzliche Anregungen und werden über die Tunnelstromerhöhung nachgewiesen.

b) Einelektronen-Tunnelbereich eU $>2$. 1 . Tunnelnde Elektronen emittieren Phononen durch Relaxation und anschließende Rekombination. 
benen charakteristischen Strom-Spannungsabhängigkeit dieses Tunnelkontaktes sei das in Abbildung 2a und b skizzierte Energie-Schema oder Halbleiterbild der Elektronenanregungen in beiden Supraleitern herangezogen. Bei den hier betrachteten Tunnelprozessen ist in diesem Schema die Gesamtenergie des Elekrrons während des Tunnelübergangs konstant. Durch die am Kontakt anliegende Spannung $U$ werden die Fermigrenzen der Supraleiter um die Energie eU (Energie in $\mathrm{eV}, \mathrm{e}=$ Elementarladung) gegeneinander verschoben. Solange diese Verschiebung kleiner als die doppelte Energielücke ist, d. h. im Bereich $\mathrm{O}<\mathrm{eU}<21$ (Abbildung 2a), kann bei der Temperatur $T=0$ kein Tunnelstrom fließen, da den unbesetzten Elektronenzuständen im Supraleiter II nur unbesetzte Elektronenzustände im Supraleiter I gegenüberstehen. Bei endlicher Temperatur $\mathrm{T}>0$ findet jedoch thermische Elektronenanregung über die Energielüke statt. Die damit verbundene Besetzung von Zuständen oberhalb der Energielücke, sowie das Auftreten von Löcherzuständen* im unteren Band führt bereits in diesem sogenannten thermischen Tunnelbereich $0<\mathrm{eU}<2 d$ zu einem geringen Tunnelstrombeitrag Jin mit der in erster Linie besetzungsbedingeten Temperaturabhängigkeit

$\mathrm{J}_{\mathrm{th}} \sim \exp (-\Delta / \mathrm{kT})$

Wird die am Tunnelkontakt anliegende Spannung über den Wert $\mathrm{eU}=2 \mathrm{~J}$ erhöht, so liegen den unbesetzten Zuständen des Supraleiters II die voll besetzten Zustände des Supraleiters I gegenüber (Abbildung 2b). Hiermit ist der steile Stromanstieg bei $\mathrm{eU}=2 \mathrm{~A}$ in Abbildung $1 \mathrm{~b}$ verbunden. Bei noch höheren Spannungen eU $\gg 2$ I nähert sich der Tunnelstrom asymptotisch einem spannungsproportionalen Verlauf. Neben dem hier beschriebenen und für die Phononendetektion und -Erzeugung wesentlichen Einelektronen- oder Einteilchen-Tunnelstrom treten noch zusätzlid andere Tunnelströme, vor allem der Josephsontunnelstrom auf.

Durch ein schwaches Magnetfeld yon etwa $1600 \mathrm{~A} / \mathrm{m}$ in der Ebene des Tunnelkon-

*Löcherzustände sind mögliche Energiezustände, die nicht von einem Elektron besetzt sind. taktes kann jedoch dieser bei den Phononenexperimenten störende Stromanteil unterdrückt werden.

\section{Phononennachweis}

Bei tiefen Temperaturen ist für eU $<21$ (Abbildung 2a) nur ein geringer thermischer Tunnelstrom zu beobachten. Treffen aus dem Innern des als Träger des Tunnelstroms dienenden Substratkristalls Phononen der Energie $E_{p h} \geqslant 2 A$ auf den Tunnelkontakt, so wird gemäß Abbildung 2a nach Absorption dieser Phononen die Zahl der angeregten Elektronen und damit der Tunnelstrom im thermischen Bereich $0<$ $\mathrm{eU}<21$ erhöht. Auf diese Weise können inkohärente Phononen der Energie $E_{p h} \geqslant$ 24 nachgewiesen werden, in Analogie zum inneren Fotoeffekt bei optischen Halbleiterdetektoren. Die durch die Energielücke bedingten Mindest-Phononenfrequenzen bzw. Energien der wichtigsten, leicht herstellbaren Supraleiter-Tunnelkontakte sind in Tabelle 1 zusammengestellt. Phononen der Energie $E_{p l t}<2.1$ besitzen demgegenüber nicht die erforderliche Energie und werden nicht nachgewiesen. Sie besitzen im Supraleiter eine um Größenordnungen längere Lebensdauer als im normalen $\mathrm{Me}$ tall. State dessen ist die Lebensdauer der Phononen mit der Energie $E_{p h}>21$ etwas kleiner als im Normalleiter.

\section{Relaxationsphononen}

Im Bereich höherer Spannungen und Tunnelströme, d.h. bei $e U \geqslant 21$ wird dem Tunnelkontakt eine beträchtliche elektrische Energie zugeführt. Unmittelbar nach dem Tunnelprozeß liegt diese Energie zunächst als Anregungsenergie der Tunnelelektronen im Supraleiter II vor, wie in

Tabelle 1. Mindestphononenfrequenzen supraleitender Kontakte.

Material $\quad \mathrm{Al} \quad \mathrm{Sn} \quad \mathrm{Pb}$

doppelte Energie-

liucke $2 \Delta$ in $\mathrm{meV} \quad 0,4 \quad 1,2 \quad 2,7$

Untere Grenz-

frequenz in $\mathrm{GHz} \quad 80 \quad 290 \quad 650$

\section{Supraleitung}

Bei gewissen Metallen, wie z. B. Zinn oder Blei, verschwindet unterhalb einer jeweils charakteristischen Temperatur $\mathrm{T}_{c}$ der elektrische Widerstand völlig. Dieses Phänomen der Supraleitung erklärt man nach Bardeen-Cooper-Schrieffer (BCS) durch die Bildung von Elektronempaaren, der sog. Cooperpaare. Die beiden Elektronen werden durch die Wechselwirkung mit dem Girter zusammengehalten. Damit besitzen sie keine weitere Wechselwirkung mit dem Gitter mehr, der Strom fließt verlustlos. Dies äußert sich in der Energieskala so, daß die Cooperpaare Energiezustände hoher Dichte besetzen, die unterhalb eines verbotenen Bereichs, dem „Gap“ liegen. Will man die Cooperpaare in ihre Bestandteile zerlegen, dann muß man sie uber das Gap anheben, ihnen also Energie zuführen. Elektronen oberhalb des Gaps zeigen keine Supraleitung mehr. Das Gap wirdbei Annäherung an $T_{a}$ immer kleiner.

Daneben ist ein Supraleiter ein idealer Diamagnet. Magnetische Felder können, wenn überhaupt, nur in Form von Feldschläuthen in das Material eindringen. Im Inneren dieser Schläuche herrscht dann Normalleitung.

\section{Tunneleffekt}

Wir wissen vom Licht, daß es dünne Metallplättchen, wie z. B. Gold, durchdringen kann, obwohl dicke Schichten absolut undurchlässig sind. Diese Eigenschaft beruht auf dem Wellencharakter des Lichtes. Barrieren in Größenordnung der Wellenlänge können abgeschwächt durchstrahlt werden. $\mathrm{Da}$ auch Materie Wellencharakter besitzt, tritt dieser Effekt dort ebenso auf; er ist als quantenmechanischer Tunneleffekt bekannt. Dünne, hohe Potentialsperren können von Teilchen kleiner Masse durchtunnelt werden. Die Tunnelwahrscheinlichkeit nimmt mit wachsender Potentialsperrenbreite und zunehmender Masse extrem stark ab. Dies ist auch der Grund dafür, daß bisher wohl noch niemand beobachtet hat, wie ein Auto eine Mauer durchtunnelt hat, ohne sie einzureißen. 
Abbildung $2 \mathrm{~b}$ gezeigt. Die Elektronen geben einen Teil ihrer Anregungsenergie durch Relaxationsübergänge zur oberen Bandkante unter Phononenemission ab.

Aus der Theorie folgt, daß das Spektrum dieser Relaxationsphononen eine scharfe Kante bei der Energiegrenze

$\mathrm{E}_{\mathrm{ph} \max }=\mathrm{eU}-2 \mathrm{~J}$

besitzt. Diesen Sachverhalt illustriert Abbildung 3a. Das Ergebnis ist unmittelbar aus der Energieerhaltung zu verstehen: Insgesamt steht für den Tunnelprozeß dic Energie eU zur Verfügung. Davon muß der Anteil 24 zum Aufbrechen eines Cooperpaares aufgewendet werden. Die Restenergie von $\mathrm{eU}-2 \mathrm{~J}$ wird auf das nach dem Tunneln im Supraleiter II vorhandene Elektron sowie auf das im Supraleiter 1 zurückbleibende Elektron aufgeteilt. In unserem Halbleiterbild entspricht dem zurüdkbleibenden Quasiteilchen eine „Lochanregung" im Supraleiter I.

Bei der Aufteilung der Überschußenergie auf die beiden Anregungen sind alle Energiewerte zwischen 0 und $\mathrm{eU}-2 \Delta$ möglich. Maximal kann ein Quasiteilchen die zusätzliche Energie eU - $2 \Delta$ erhalten, während das andere Quasiteilchen keine Überschußenergie erhält. Für die Relaxation eines injizierten Quasiteilchens zur oberen Bandkante steht dementsprechend höchstens die Energie eU - $2 \Delta$ zur Verfügung.

\section{Rekombinationsphononen}

Im Anschluß an die Relaxation können die Quasiteildhen in den Grundzustand rekombinieren. Dies entspricht in Abbildung 2b einem Ubergang von der oberen zur unteren Bandkante (vergleiche ElektronLoch Rekombination). Die anfallende Energie wird als Rekombinationsphonon mit der Energie $E_{p h}=2 \Delta$ abgestrahlt. Die Energie der Rekombinationsphononen ist im Gegensatz zum Spektrum der Relaxationsphononen von der am Tunnelkontakt anliegenden Spannung $U$ in erster Näherung unabhängig.

\section{Experimente}

Bei Experimenten zur Phononenemission und -detektion mit supraleitenden Tunnelkontakten werden die Tunnelkontakte (Schichtdicken etwa $1000 \AA$ ) auf den ge- genüberliegenden, sehr gut polierten Oberflächen eines Isolator-Einkristalls, z. B. aus Korund, $\mathrm{Al}_{2} \mathrm{O}_{3}$ (Saphir) oder auch hochreinem Silizium bzw. Germanium, hergestellt. Diese Kristalle erfüllen, wie man aus Wärmeleistungsmessungen weiß, die Voraussetzung einer genügend langen Phononenlebensdauer bei Temperaturen von 1-4 K, d.h. im Temperaturbereich der Supraleitung. Die in Abbildung 4 gezeigre grundsätzliche Anordnung befindet sich daher bei den Experimenten im Innern eines He-Kyrostaten, dessen Temperatur zwischen 1 und $2 \mathrm{~K}$ durch Abpumpen des Heliumdampfes variiert werden kann. Bei Anlegen einer Pulsspannung an den Generatortunnelkontakt werden Phononen emittiert, die nach einer Laufzeit t den Detektor erreichen, dort durch Spaltung von Cooperpaaren absorbiert werden und den Tunnelstrom des im nthermischen" Arbeitsbereich betriebenen Detektors erhöhen. Das Oszillogramm in Abbildung 5 zeigt bei einem Siliziumkristall als Substrat mit SnI-Sn Generator und Detektor deutlich drei verschiedene Phononsignale. Die Laufzeiten $t_{1}$, ta der beiden ersten Signale entsprehen der Ausbreitung einer mechanischen Longitudinalwelle und einer Transversalwelle. Das dritte und vierte Signal sind einer Longitudinal- und einer Transversalwelle zuzuordnen, die an den zylindrischen Seitenwänden des Kristalls gestreut werden und erst danach den Detektor erreichen.

Dieses Ergebnis zeigt wegen der Ubereinstimmung mit der aus der Schaligeschwindigkeit $\left(t=d / c_{s} ; d=\right.$ Schichtdicke; $c_{s}=$ Schallgeschwindigkeit) berechneten Phononenlaufzeit eindeutig, daß die beobachteten Signale auf Phononen zurüdkzuführen sind. Außerdem deutet die Reflexion an der Seitenwand auf eine allseitige Phononenabstrahlung durch den Generator, in Ubereinstimmung mit der Erwartung einer inkohärenten Phononenentstehung. Bei kohärenter Phononenerzeugung, z. B. in Form einer ebenen Welle, würde man statt dessen eine scharfe Bündelung der Phononen in bestimmten Raumrichtungen erwarten.

Die für die vorher geschilderten Phononenprozesse wesentliche Frage nach der Frequenz der abgestrahlten Phononen kann am einfachsten durch die Abhängigkeit des detektierten Phononensignals von der Spannung des Generatortunnelkontakts überprüft werden.
Bei übereinstimmenden Energielücken ist das Detektorsignal proportional zur Zahl der vom Generator abgestrahlten Rekombinationsphononen sowie proportional zur $\mathrm{Zahl}$ der abgestrahlten Relaxationsphononen, falls letztere eine Energie $E_{p h} \geqq 2 \Delta$ besitzen. Mit der oberen Frequenzgrenze der Relaxationsphononen von $E_{\mathrm{ph} \max }=\mathrm{eU}-2 \Delta$ folgt, daß Relaxationsphononen erst ab einer Generatorspannung $\mathrm{eU} \geq 4 \Delta$ beobachtet werden können.

Dieses Verhalten wird durch die Messung der Detektorsignalamplitude in Abhängigkeit vom Sendestrom bzw. der Sendespannung (vergl. Abbildung 6a) bestätigt: Der bis zur Generatorspannung $4 \Delta$ (abgesehen von geringen Abweichungen) stromproportionale Verlauf des Detektorsignals ist verständlich, da die Abstrahlung der Rekombinationsphononen proportional zum $\mathrm{Ge}$ neratorstrom sein sollte. Der bei $\mathrm{eU}=4 \Delta$ auftretende Knick in der Steigung entsteht durch den zusätzlichen Beitrag der Relaxationsphononen. Bis zur Spannung $6 \Delta$ gilt ebenso ein nahezu linearer Signal-Sendestrom-Zusammenhang. Oberhalb der Spannung von $6 \Delta$ tritt ein zusätzlicher parabelförmig zunehmender Signalanteil auf, der durch Wiederabsorption von Relaxationsphononen durch Anregung von Quasiteilchen (Paarbrechen) und anschließende Reemission (als zusätzliche Rekombinationsphononen) gedeutet werden kann. Relaxationsphononen der Energie $E_{p h} \geqq 4 \Delta$ spalten hierbei in 2 Phononen der Energie $E_{p h} \geqq 2 \Delta$ auf, so daß die Anzahl der insgesamt nachgewiesenen Phononen größer ist als die Anzahl der primär erzeugten Relaxationsphononen.

Eine genauere Analyse des Zusammenhanges zwischen Detektorsignal und Generatorstrom bzw. Generatorspannung ist durch Bildung der ersten Ableitung $\operatorname{di}_{D}\left(i_{G}\right) / d_{i}$ des Detektorstromes in nach dem Generatorstrom if möglich. Experimentell wird hierzu der Generator mit Gleichstrom bei Oberlagerung kleiner Gleichstrompulse oder eines Sinuswechselstromes geringer Amplitude betrieben. Das Detektorsignal (Puls- bzw. Sinusanteil) entspricht unter diesen Bedingungen der Ableitung din $/ \mathrm{di}_{i}$, wobei im Falle der Oberlagerung eines Sinuswechselstromes die Methode der phasenempfindlichen Gleichrichtung zu wesentlicher Verbesserung des Signal-Rauschverhältnisses führt. Das Ergebnis der Messung ist in Abbildung $6 \mathrm{~b}$ mit der unter Berück- 

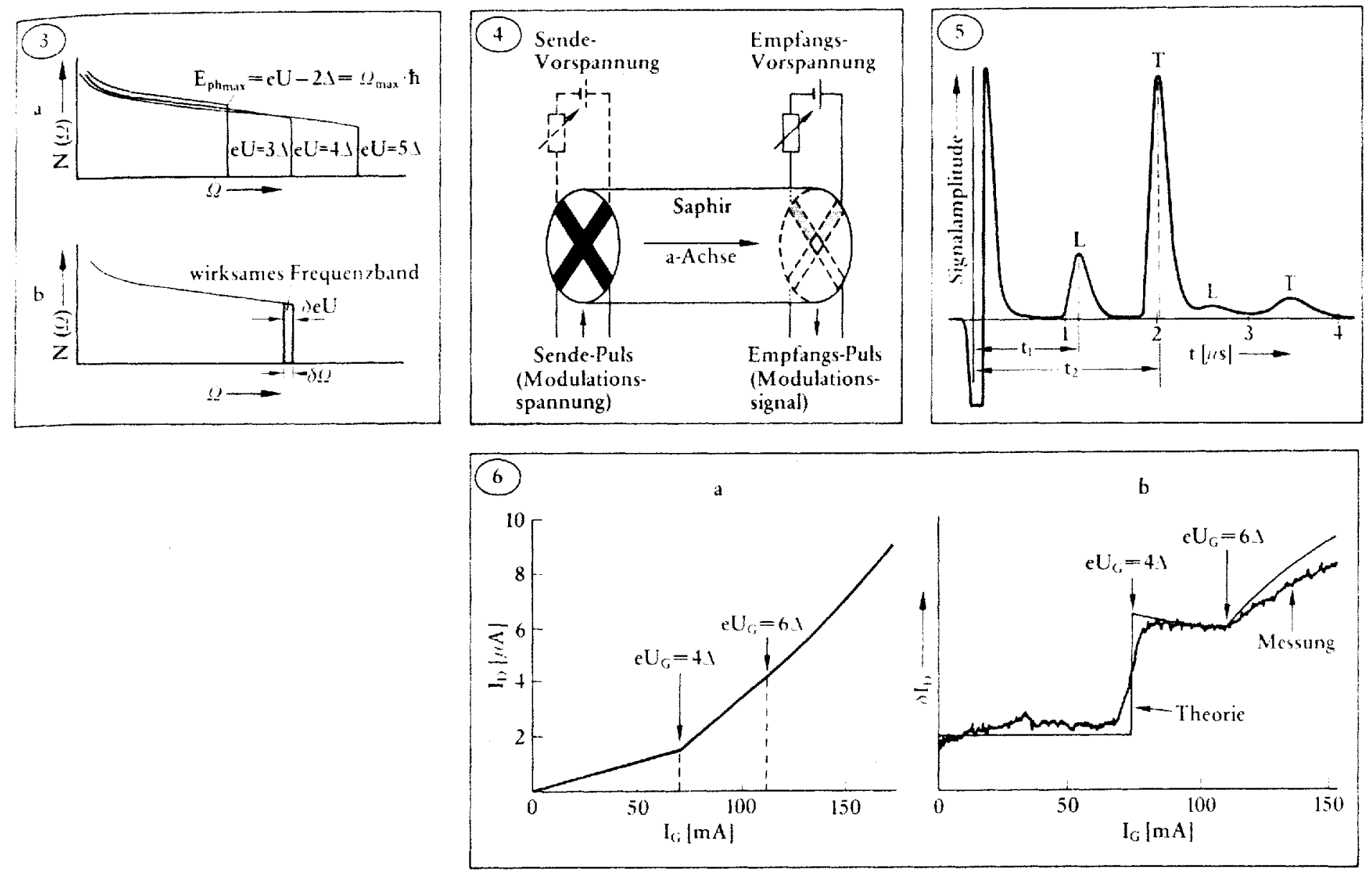

Abb. 3. a) Berechnetes Relaxationsspektrum für verschiedene Spannungen $U$.

b) Anderung des Phononenspektrums bei einer kleinen Spannungsänderung $\delta U$. Die Anderung des Phononenspektrums erfolgt vorwiegend bei der Maximalenergie eU 24. Hieraus folgt, daß ein schmales Phononenband durch Modulation (schraffiert) aus dem Gesamtspektrum separiert werden kann.

Abb. 4. Experimentelle Anordnung der Kristallprobe mit Tunnelkontakten zur Phononenerzeugung und zum Phononennachweis nach Eisenmenger und Dayem. Die Sendevorspannung wird im Modulationsexperiment benötigt.

Abb. 5. Empfangene Phononenpulse mit Si-Substrat, Detektor und Generatortunnelkontakt Sn-I-Sn. L = Longitudinale Phononen in [111]-Richtung mit $t_{1}=1,1$ us Verzögerungszeit (Kristalldicke $0,9 \mathrm{~cm}$ ) $\mathrm{T}=$ transversale Phononen mit $\mathrm{t}_{2}=2,0 \mathrm{us}$ Verzögerungszeit, $\mathrm{L}^{\prime}=$ Longitudinale Phononen nach Streuung an der Seitenwand, $\mathrm{T}^{\prime}=$ Transversale Phononen nach Streuung an der Seitenwand.
Abb. 6. a) Detektorsignal als Funktion des Generatorstroms mit der Angabe der zugehörigen Generatorspannungen $\mathrm{eU}=4 \mathrm{~A}$; 61. Bei der Generatorspannung $41 \mathrm{bc}$ ginnt der Signalbeitrag der Relaxationsphononen. b) Detektorsignal differenziert nach dem Generatorstrom (als Funktion des Generatorstroms). Vergleich mit dem theoretischen Verlauf. sichtigung des theoretischen Phononenspektrums sowie unter Einschluß der Wiederabsorption berechneten Signalabhängigkeit wiedergegeben. Die vorhandenen Abwejchungen, besonders das Maximum im Spannungsbereich $2 \Delta<\mathrm{eU}<4 \Delta$ ist auf den Einfluß der endlichen Meßremperatur zurïk zuführen, wobei unter anderem die direkte Rekombination von injizierten Quasiteilchen mit thermisch angeregten Quasiteilchen ohne vorherige Relaxation berüdssidntigt werden muß.

Hier warten noch Feinheiten der Spannungs- und Temperaturabhängigkeit des abgestrahlten Phononenspektrums und damit im Zusammenhang Fragen der Elektron-Phononwechselwirkung im Supraleileiter auf eine endgültige Klärung. Die beschriebenen wesentlichen Merkmale des Relaxations-und Rekombinationsspektrums können jedoch durch diese und die folgenden experimentellen Ergebnisse als gesicher: gelten.

\section{Bestimmung von Emissionsspektren}

Die bisher beschriebenen Experimente stehen zwar im Einklang mit der Vorstellung, 
da $\Omega$ ein schmalbandiges Rekombinationsspektrum und ein breitbandiges Relaxationsspektrum auftreten, licfern jedoch nicht die genaue spektrale Verteilung der Phononen. Die Analyse des emittierten Phononenspektrums erfordert grundsätzlich Methoden, wie sie auch aus der Optik bekannt sind. Es können abstimmbare, schmalbandige Detektoren nach Renk*, Interferenzfilteranordnungen (Blackford), sowie auch schmalbandige "Absorptionsfilter ${ }^{\alpha}$ verwendet werden.

Das zuletzt genannte Prinzip wurde von Narayanamurti und Varma unter Benutzung resonanter elektronischer Übergänge von Antimondonatoren in Germanium zur Analyse des von einem Sn-I-Sn Tunnelkontakt abgestrahlten Rekombinations-Phononenspektrums verwirklicht. Je ein $\mathrm{Sn}-\mathrm{I}-\mathrm{Sn}_{\mathrm{n}}$ Tunnelkontakt als Phononengenerator und -detektor befinden sich - wie Abbildung 7a zeigt - auf den gegenüberliegenden Grenzflächen [110] des mit Sb dotierten Ge-Einkristalls.

Die bereits durch das Kristallfeld vorhandene Aufspaltung des wasserstoffähnlicten Elektronengrundzustandes des flachen Donators $\mathrm{Sb}$ kann durch eine einachsige mechanische Spannung [111] im Bereich von $10^{11} \mathrm{~Hz}$ bis $5 \cdot 10^{11} \mathrm{~Hz}$ (vergl. Abbildung $7 b$ ) weiter erhöht werden. Zwischen den ausgezogenen Niveaus sind resonante Phononabsorptionsübergänge mit Ausbreitung in [110]-Richtung erlaubt. Das Detektorsignal als Funktion des Drucks (Abbildung 7i) zeigt bei der mit der Frequenz der Rekombinationsphononen übereinstimmenden $\mathrm{Ni}$ veauaufspaltung von $2,9 \cdot 10^{11} \mathrm{~Hz}$ ein ausgeprägtes Minimum. Damit wird bestätigt, daß der wesentliche Phonon-Signalbeitrag in diesem Experiment auf die Ausbreitung von Rekombinationsphononen zurückgeht.

Das Experiment bestätigt außerdem die aus der Energieluicke berectnete Frequenz der Rekombinationsphononen durch Anschlus an die ferninfrarot-optische Bestimmung der Niveauaufspaltung der Sb-Störstelle. Eine Auflösung der tatsächlichen Linienform und Breite der Rekombinationsphononen gelang u. a. als Folge von Druckinhomogenitäten noch nicht.

"Siehe "Physik in unserer Zeir ${ }^{\alpha} 1,16$ (1974).
Eine weitere, noch direktere Bestätigung der Frequenz und Schmalbandigkeit der Rekombinationsphononen gelang Bladkford mit Al-Tunnelkontakten als Generator und Detektor. In diesem Experiment wurde ein Helium-Film, dessen Dicke durch Einstellung des Dampfdruckes kontinuierlich verändert werden konnte, als angekoppeltes Phononenresonanzsystem verwendet. Im Helium-Film treten Interferenzen longitudinaler Phononen auf, die bei bestimmten Resonanzdicken zu einer besonders starken Dämpfung der Phononen (Ultraschallwellen) führen. Aus der diesen Schichtdicken zugeordneten Wellenlänge kann nach den Zusammenhängen

$\mathrm{d}_{\text {Resonan } 2}=\lambda / 4+n \lambda / 2$ mit $n=1,2,3 \ldots$

$\left(\lambda=\right.$ Phononenwellenlänge, sowie $\mathrm{f}=\mathrm{c}_{\mathrm{s}} / \lambda$, $\mathrm{f}=$ Frequenz,$c_{\xi}=$ Schallgeschwindigkeit in Helium) die Phononfrequenz $f$ berechnet werden. Auch hier ergab sich Übereinstimmung mit der aus der Energielücke folgenden Frequenz der Rekombinationsphononen.

\section{Anwendungen zur Spektroskopie mit Phononen}

Die Spektroskopie mit Phononen, d. h. die Bestimmung einer frequenzabhängigen $A b$ sorption von Phononen, wie sie beispielsweise an Störstellen und Fremdatomen in Eink ristallen auftreten, setzt eine kontinuierlich abstimmbare schmalbandige Phononenquelle voraus, wobei der Phononendetektor möglichst breitbandige Eigenschaften besitzen sollte.

\section{Spektroskopie mit Rekombinations-} phononen

Erste Versuche, das Spektrum der Rekombinationsphononen durch Beeinflussung der Energielücke zu verändern, wurden ron Narayanamurti und Mitarbeitern unternommen. Durch Anlegen eines Magnetfeldes parallel zur Ebene der dünnen $\mathrm{Sn}$ Schichten eines Tunnelkontaktes kann die Energielücke erniedrigt werden. Allerdings ist mit dieser Erniedrigung auch eine Verbreiterung des Rekombinationsspektrums und ein Verlust an spektraler Auflösung verbunden. Grundsätzlich kann die Energielücke auch durch Erhöhung der Temperatur herabgesetzt werden. Dies hat allerdings auf der Detektorseite einen ganz erheblichen Empfindlichkeitsverlust zur Folge.
Abb. 7. Bestimmung der Frequenz der Rekombinationsphononen durch die druckabhängige Resonanzabsorption von $\mathrm{Sb}$ in Ge. a) Anordnung $\mathrm{Sn}-\mathrm{I}-\mathrm{Sn}$ Generator und Detektor; b) Niveauaufspaltung von $\mathrm{Sb}$ in $\mathrm{Ge}$ durch einachsige mechanische Normalspannung; c) Phononensignal in Abhängigkeit vom Drudk.

Abb. 8. Modulationsspektroskopie der Phononenresonanzstreuung in $\mathrm{Al}_{2} \mathrm{O}_{3}: \mathrm{V}^{3+}$; Meßkurve: Signalanteil der „Relaxationsphononenlinie" bei eU-2 $\Delta$ mit der durch $\mathrm{V}^{3+}$ verursachten Absorption; horizontale gestrichelte Linie: konstanter Signalbeitrag durch Rekombinationsphononen des Sn-ISn-Senders.

Abb. 9. Phononenresonanzstreuung von Sauerstoff in Si, Kurve A=erste Ableitung des Detektorsignals nach der Generatorspannung; Kurve $\mathbf{b}=\mathbf{z w e i t e}$ Ableitung des Detektorsignals nach der Generatorspannung. 

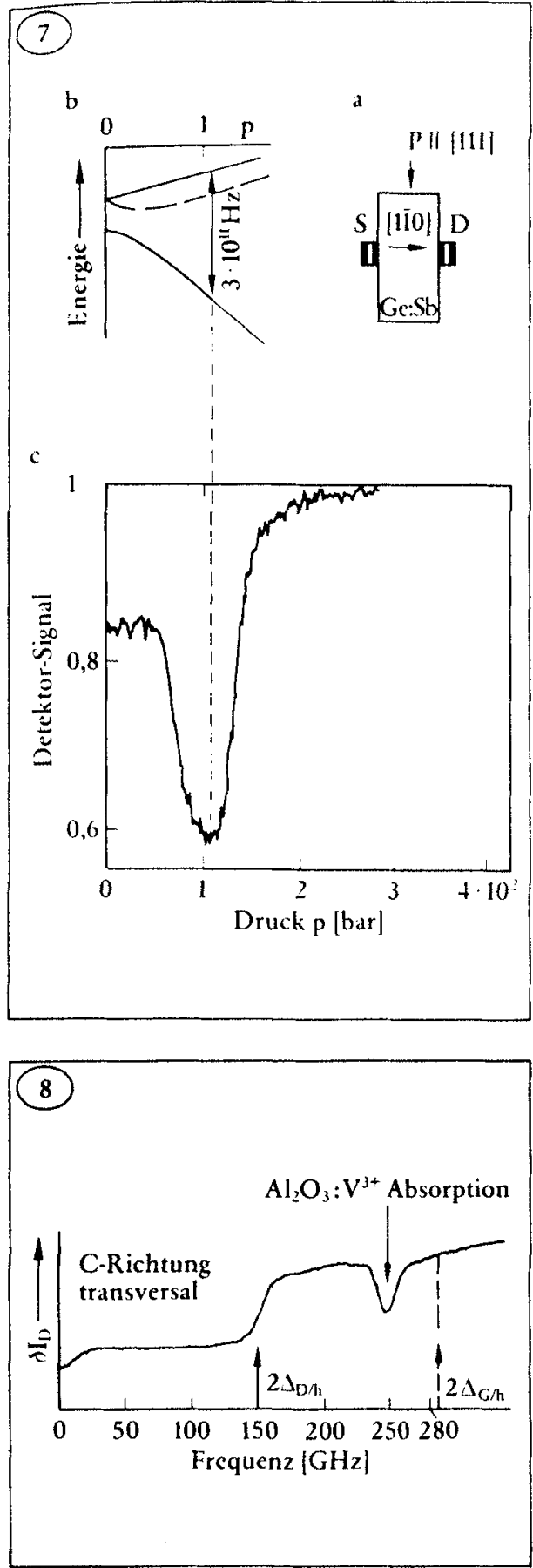

Spektroskopie mit Relaxationsphononen

Das Spektrum der Relaxationsphononen ist gegenüber dem Spektrum der Rekombinationsphononen breitbandig, besitzt jedoch eine scharfe obere Grenzfrequenz, die leicht über die Batteriespannung eingestellt werden kann. Mit Hilfe elektronischer Modulationstechniken ist es nun möglich, diese Eigenschaft des Relaxationsspektrums für Phononenabsorptionsmessungen auszunutzen, so als ob eine schmalbandige Phononenquelle mit veränderlicher Frequenz zur Verfügung stünde.

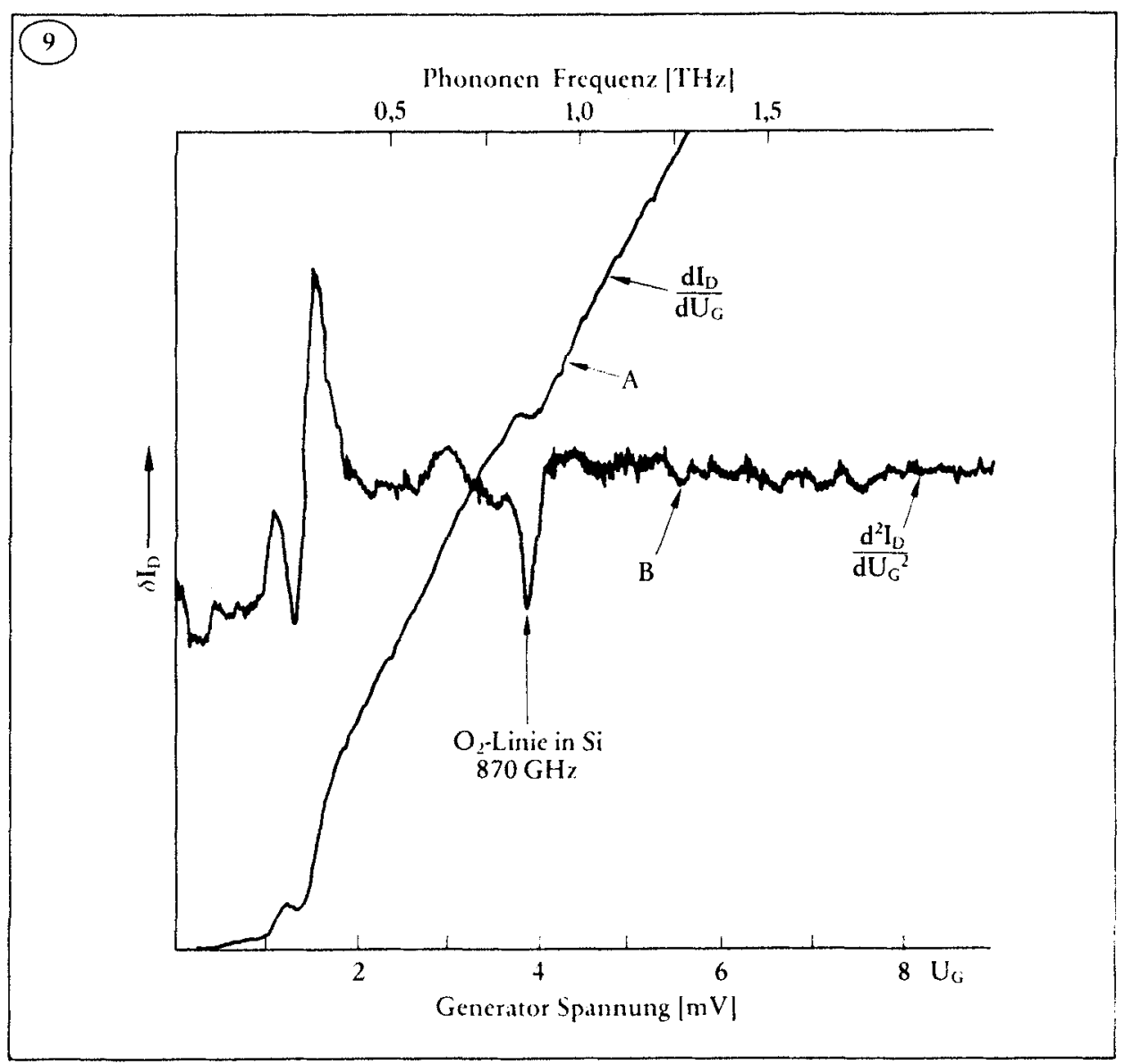

Der bei der oberen Grenzenergie $\mathrm{E}_{\mathrm{ph} \max }=$ eU $-2 \Delta$ nahezu rechtedkförmige Spektralverlauf ergibt, (Abbildung 3b) bei Modulation mit einer kleinen Wechselspannung $\delta U$, nur Spektraländerungen in Form einer scharfen Phononenlinie der Breite $\delta \mathrm{eU}$ an der Stelle $E_{p}=e U-2 \Delta$. Bei der Messung wird jetzt daher nur der Modulationsanteil des Detektorsignals benutzt. Dieses von Kinder erstmalig angewandte Prinzip der Phononenspektroskopie zeigte am System $\mathrm{V}^{3+}$ in $\mathrm{Al}_{2} \mathrm{O}_{3}$ eine mit Ferninfrarotmessungen vergleichbare hohe Auflösung der scharfen Absorptionslinie, Abbildung 8.

Bei diesem Experiment wurde ein Sn-I-SnTunnelkontakt als Phononengenerator und ein Al-I-Al-Tunnelkontakt als Detektor benutzt. Da der Al-Detektor eine kleinere Energielücke besitzt als der Sn-Generator, können damit Relaxationsphononen im Energieintervall $2 \Delta_{\mathrm{Al}}$ bis $2 \Delta_{\mathrm{Sn}}(150 \mathrm{GHz}-$ $280 \mathrm{GHz}$ ) nachgewiesen werden. Phononen unterschiedlicher Polarisation und Richtung werden verschieden stark absorbiert und die so erhaltenen Absorptionsquerschnitte können mit theoretischen Ergebnissen (Auswahlregeln) verglichen werden. Kinder gelang eine experimentelle Trennung der Si- gnalanteile verschiedener Polarisation durch Ausnutzung der Laufzeitunterschiede der Longitudinal- und Transversal-Impulse.

Da die maximale Energie der primär ent stehenden Relaxationsphononen nur durch: die Batteriespannung bestimmt ist, sollte eine Spektroskopie mit Relaxationsphononen grundsätzlich bis zur Gittergrenzfre quenz des Generators möglich sein. Das Experiment zeigt jedoch, daß zumindest in Zinn und Blei als Supraleiter die Weglängø für Phononen, deren Energie größer als dic Energielücke ist, kleiner wird als die Schichtdicke der Tunnelkontakte. Dies entspricht der Vorstellung, daß die Phononenabsorption, die ja im Detektor einen Phononennachweis durch Aufbrechen von Cooperpaaren ermöglicht, bereits ebenso im Generator bei der Phononenenergie $E_{p h}$ $2 \Delta$ Generator $z$ u einer starken Wiederabsorp tion führt. Primäre Relaxationsphononen deren Energie höher ist als $2 \Delta_{\text {Generator }}$, werden daher, bevor sie die Generatorschich verlassen können, unter Paarbrechen absor biert und verlieren ihre Energie.

Im Experiment nach Abbildung 5 ist dahe die obere Energiegrenze $E_{p}=2 \Delta_{\text {Generato }}$ 
und der gesamte verfügbare spektrale Bereich $2 \Delta_{\text {Detektor }}<\mathrm{E}_{\mathrm{p}}<2 \Delta_{\text {Generator. }}$.

Eine Herabsetzung der störenden Wiederabsorption gelingt nach Welte et. al. durch Verwendung von Al-Tunnelkontakten als Phonongenerator, da die Elektron-Phonon-Wechselwirkung in $\mathrm{Al}$ im Einklang mit der niedrigen Energielücke schwächer ist als in $\mathrm{Sn}$ und $\mathrm{Pb}$. Man kann leicht prüfen, ob hochenergetische Relaxationsphononen mit $E_{p}>2 \Delta_{\text {Generator }}$ abgestrahlt werden, wenn bei Al-I-Al als Generator eine $\mathrm{Sn}-\mathrm{I}-\mathrm{S} n$-Tunneldiode als Detektor verwendet wird. Erst bei einer Mindestbatteriespannung $e U_{\mathrm{m}} \geq 2 \Lambda_{\text {Gentrator }}$ $+2 \Delta_{\text {Detektor }}$ besitzen die Relaxationsphononen eine Energie $E_{p} \geqq 2 \Delta_{\text {Detektor }}$. Wegen $2 \Delta_{\text {Detektor }}>2 \Delta_{\text {Generator }}$ ist die Beobachtung eines Signaleinsatzes bei der Mindestbatteriespannung $U_{n}$ der direkte Beweis für die Abstrahlung von Relaxationsphononen mit $E_{p} \geqq 2 \Delta_{\text {Generator. }}$ Im Experiment wurden auf diese Weise mit einem Sn-Detektor Phononen bei $3 \cdot 10^{11} \mathrm{~Hz}$ beobachtet, die aus einem Al-Sender (Energielüdke liegt bei $10^{11} \mathrm{~Hz}$ ) emittiert wurden.

Darüber hinaus konnten Forkel et. al. zeigen, daß Al-Tunnelkontakte selbst im Bereich nahe bei $10^{12} \mathrm{~Hz}$ bei entsprechend hoher Batteriespannung U Relaxationsphononen gemäß der Bedingung $E_{p \max }=e U-2 \Lambda$. theratar abstrahlen. Zur Frequenzkontrolle wurde das Siliziumsubstrat, in das sich die Phononen ausbreiten, mit Sauerstoff dotiert. Aus der Ferninfrarotabsorption ist bekannt, daß durch lokalisierte Schwingungen des Sauerstoffs ein scharfer Ubergang bei $870 \mathrm{GHz}$ (entsprechend $3,9 \mathrm{meV}$ oder $29 \mathrm{~cm}^{-1}$ ) auftritt.

Diese Resonanzstreuung wurde nun auch im Phononenexperiment ( $\mathrm{Al}$ als Generator, $\mathrm{Sn}$ als Detektor), Abbildung 9, als deutliche Struktur bei der entsprechenden Batteriespannung gefunden. Bei der Messung Kurve $\mathrm{A}$ in Abbildung 9 wurde äbnlich wie bei der Messung von Kinder (Abbildung 6) die Generatorspannung $U$ mit einer additiven kleinen Sinusspannung $\delta \mathrm{U}$ moduliert. Ais Signal ist wieder nur der resultierende Modulationsanteil der Detektorspannung aufsetragen. Bei der Phononenresonanz des Sauerstoffs wird bei dieser Messung anstelle eines ausgeprägten Minimums im Bereich der Resonanzspannung ein kurzer, nahezu konstanter Verlauf im sonst stetigen Signalanstieg beobachtet.
Dieses Ergebnis steht im Einklang mit theoretischen Ergebnissen, die bei hohen Generatorspannungen eU $\gg 2 \Delta_{G \text { enerator, }}$ vergl. Abbildung 3a, nur noch eine geringe Höhe der scharfen Stufe des Relaxationsspektrums bei der Maximalenergie $E_{p \max }=$ eU $-2 \Delta_{\text {Generator }}$ voraussagen. Das Spektrum der Relaxationsphononen geht im Grenzfall sehr boher Generatorspannungen in den für Normalleiter erwarteten Verlauf mit verschwindender Stufenhöhe über.

Da die Modulation der Generatorspannung im Experiment der Bildung der ersten Ableitung des Relaxationsspektrums entspricht, wird bei endlicher Stufenhöhe hierdurch eine scharfe Phononenlinie bei $E_{p}=e U-$ $2 \Delta_{\text {Generator }}$ erzeugt. Demgegenüber liefert bei einem Relaxationsspektrum mit verschwindender Stufe und stetigem (linearen) Anstieg nach tieferen Frequenzen erst die zweite Ableitung nach der Generatorspannung eine scharfe Spektrallinie. Diese Verhältnisse liegen bei der Messung Abbildung 9, Kurve B, vor.

Im Experiment wird die zweite Ableitung (bei Sinusmodulation der Generatorspannung) aus der zweiten Harmonischen des Detektormodulationssignals gewonnen. Das entsprechende Meßresultat in Abbildung 9, Kurve $B$, zeigt nun, wie erwartet, die Absorptionslinie der Resonanzstreuung des Sauerstoffs. Die erzielte Auflösung ist bei dieser Messung noch zum Teil durch den Einflußs der endlichen Temperatur auf das Phononenspektrum des Generators begrenzt. Hierdurch wird neben dem Signaleinsatz bei $\mathrm{eU}=2 \Delta_{\mathrm{Generatur}}+2 \Delta_{\text {jotehtir }}$ in Abbildung 9, Kurve A, ein zusätzlicher Signalbeitrag bei $\mathrm{eU}=2 \Delta_{\mathrm{Generater}}$ hervorgerufen. Auch in Kurve B treten bei diesen Generatorspannungen entsprechend starke Strukturen auf.

Die Beobachtungen der $\mathrm{V}^{3}{ }^{+}$-Resonanz in $\mathrm{Al}_{2} \mathrm{O}_{3}$ und der Sauerstoff resenanz in Silizium lassen es sinnroll erschinen, ahhnliche Untersuchungen bei noch höheren Phononenenergien durchzuführen, um weitere Aufschlüsse über das abgestrahlte Phononenspektrum zu erhalten und zu prüfen, bis zu welcher oberen Frequenzgrenze Supraleitertunneldioden für eine Absorptionsspektroskopie mit akustischen Phononen geeignet sind.

\section{Literatur}

W. Eisenmenger und A. H. Dayem, Phys. Rev. Letters 18, 125 (1967).

V. Narayanamurti and C. M. Verma, Phys. Rev. Letters 25, 1105 (1970).

B. L. Blackford, Phys. Rev. Letters 28, 414 (1972).

H. Kinder, Phys. Rev, Letters 28, 1564 (1972).

M. Welte, K. Lassmann und W. Eisenmenger, Journal de Physique, Colloque C4, supplément au n ${ }^{\circ} 10$, Oct. 1972, S. C4-25.

W. Forkel, M. Welte und W. Eisenmenger, Phys. Rev. Letters 31, 215 (1973).

W. Eisenmenger, geboren am 11. Februar 1930 in Gelsenkirchen, 1950-1955: Phy sikstudium an der TH Aachen und an der Universität Göttingen, ab 1952 im 3. Physikalischen Institut der Universität Güttingen bei Prof. Erwin Meyer, dort 1958 Promotion. Ab 1959: Wissenschaftlicher Assistent bei Prof. Erwin Meyer, 1964 Habilitation im Fach Physik. Ab 1964 Universitätsdozent am 3. Physikalischen Institut der Universität Göttingen. 1966:67 Aufenthalt bei den Bell Telephone Laboratories, Murray Hill, USA. Seit 1969 ordentliche Professur an der Universität Stuttgart, Lehrstuhl für Experimentalphysik. 